\title{
Untersuchungen zur Schlachtkörper- und Fleischqualität mit Hilfe von MR-Tomographie und MR-Spektroskopie
}

\author{
Herrn Professor Dr. Erhard Kallweit zum 65, Geburtstag gewidmet
}

\begin{abstract}
Summary
Title of the paper: Studies of carcass and meat quality by means of MR tomography and MR spectroscopy Magnetic resonance tomography (MRT) is an important diagnostic tool, originally developed for imaging in human medicine. In animal science it has been applied primarily to determine body composition in live animals and in carcasses. Studies in pigs, sheep and waterfowl indicate that quantification of soft tissue by means of prediction equations or by direct measurement of lean and fat volume is highly accurate. So MRT is particularly suitable to analyse growth and development of animals regarding to quality safety in meat production. A precise discrimination between soft tissues enables this technique to serve as a reference for the improvement of systems used in practical animal breeding and carcass grading. Based on the same principle magnetic resonance spectroscopy (MRS) offers a new possibility to investigate muscle physiology in live animals. The energy metabolism of muscle in pigs with different MHS genotype was studied by means of MRS regarding to an optimum meat quality.
\end{abstract}

Key Words: body composition, percentage of lean, growth, meat quality, stress susceptibility, MHS genotype, magnetic resonance

\section{Zusammenfassung}

Die Magnet-Resonanz-Tomographie (MRT) wurde fur die Humanmedizin entwickelt und ist dort eines der wichtigsten bildgebenden Diagnoseverfahren. In der Tierzuchtforschung wurde die MRT hauptsächlich zur Bestimmung der geweblichen Zusammensetzung von lebenden Tieren und Schlachtköpern eingesetzt, In Untersuchungen an Schweinen, Schafen und Wassergeflugel wurde belegt, dass die Quantifizierung von Weichgeweben entweder uber Schätzgleichungen oder eine direkte Erfassung des Muskel- und Fettvolumens mit sehr hoher Genauigkeit moglich ist. Damit ist das Verfahren besonders fur Studien zu Wachstum und Entwicklung von Tieren als einem Kriterium der Qualitătssicherung bei der Fleischerzeugung geeignet. Die exakte Differenzierung zwischen Weichgeweben lässt einen Einsatz als Referenzmethode bei der Weiterentwicklung von Verfahren fur die tierzuchterische Praxis und Schlachtkorperklassifizierung zu. Auf́ demselben Grundprinzip beruht die Magnet-Resonanz-Spektroskopie (MRS), ein Verfahren, das neue Einblicke in die Muskelphysiologie des lebenden Tieres ermöglicht. Der Energiestoffwechsel in der Muskulatur von Schweinen mit unterschiedlichem MHS-Genotyp wurde im Hinblick auf eine optimale Fleischbeschaffenheit mit Hilfe der MRS untersucht.

Schlusselworter: Korperzusammensetzung, Fleischanteil, Wachstum, Fleischbeschaffenheit, Stressernpfindlichkeit, MHS-Genotyp, Magnet-Resonanz

\section{Einleitung}

Für eine ausgewogene und gesunde menschliche Ernährung ist Fleisch ein wichtiger Bestandieil, da es sowohl in ernährungsphysiologischer als auch in sensorischer Hinsicht eine hohe Qualităt aufweist. Diese Qualităt ist das Ergebnis einer Reihe physiolo- 
gischer Vorgänge und morphologischer Entwicklungen im Organismus des lebenden Tieres. Bisher wurde für die Qualitätssicherung bzw. für die Verbesserung der Produktqualität hauptsächlich eine Endproduktkontrolle eingesetzt; diese wird jedoch zunehmend durch ein System der präventiven Prozesskontrolle ersetzt. Dazu wird der Analysezeitpunkt einzelner Einflussfaktoren immer weiter vorgezogen, so dass die Abläufe im lebenden Tier als Steuermechanismen für die sich daraus ergebende Produktqualität in den Mittelpunkt des Interesses gerückt sind.

Eine intensivere Beachtung gilt dem Erzeugungsprozess aber auch, weil für eine steigende Zahl von Verbrauchern zur Qualität des tierischen Erzeugnisses auch die Herkunft, Aufzucht und Haltung der Tiere zählt. Zuchtziele müssen zukünftig vermehrt über ganzheitliche Betrachtungsweisen definiert werden, wozu u. a. biologisch fundierte Wachstumsmodelle eine Grundlage bilden. Den Erfordernissen einer nachhaltigen Landwirtschaft kann so Rechnung getragen werden. Hieraus resultiert die hohe Bedeutung von in vivo Untersuchungen zu Wachstum und Entwicklung bei landwirtschaftlichen Nutztieren. In der Tierzuchtforschung haben vor diesem Hintergrund in den letzten Jahren vor allem nicht invasive bildgebende Messtechniken, wie die Röntgen-Computertomographie und die Magnet-Resonanz-Tomographie besondere $\mathrm{Be}$ deutung erlangt (KALLWEIT et al., 1994; SZABO et al., 1999; VANGEN und JOPSON, 1996).

Im Institut für Tierzucht und Tierverhalten der FAL wird die Magnet-Resonanz-Tomographie seit über zehn Jahren zur Bestimmung der Körperzusammensetzung, für Analysen des Gewebewachstums und fur anatomisch morphologische Studien an landwirtschaftlichen Nutztieren eingesetzt. Mit der Magnet-Resonanz-Spektroskopie steht darüber hinaus eine Technik zur Verfügung, die zur Untersuchung des PhosphorStoffwechsels in Muskulatur und Organen am lebenden Organismus geeignet ist. Die Themenkomplexe Morphologie, Wachstum und Muskelphysiologie sind wichtige Bestandteile der Forschung in der Kette „Wachstum - Schlachtkörper- und Fleischqualität".

2.

MR-Tomographie und MR-Spektroskopie

Die seit Mitte der achtziger Jahre in der Humanmedizin eingesetzte Magnet-ResonanzTomographie (MRT) zählt dort mittlerweile zu den bedeutendsten Diagnoseverfahren. Mit Hilfe dieser auch unter dem Namen Kernspintomographie bekannten Technik lassen sich Schnittbilder des Körpers in beliebiger Orientierung und von nahezu jeder gewünschten Körperpartie erzeugen. Diese Aufnahmen zeichnen sich vor allem durch eine äußerst kontrastreiche Differenzierung von Weichgeweben aus. Die MRT basiert auf magnetischen Eigenschaften von Wasserstoffatomkernen (Protonen), die im Körper in großer Zahl vorhanden sind. Durch die Einstrahlung von Radiowellen einer bestimmten Wellenlänge in Form von Pulssequenzen werden die Protonen veranlasst, ihrerseits elektromagnetische Wellen abzugeben, die charakteristisch für verschiedene Gewebe sind. Diese Radiowellen werden von einer Empfangsspule aufgenommen, d.h. es werden zeitlich abnehmende Signale induziert, die schließlich von einem Computer in ein Bild umgesetzt werden. Durch wiederholte Messungen während dieses freien Induktionsabfalls können sog. Echos ausgelesen werden, die die Rückkehr der Proto- 
nen in das energetische Gleichgewicht beschreiben. Unter Anwendung spezieller Methoden der Bildauswertung lassen sich exakte Aussagen über den Muskel- und Fettgehait des Körpers treffen.

Werden statt der Wasserstoffprotonen Phosphoratomkerne im Magnetfeld angeregt, ist die nicht invasive Untersuchung des Phosphorstoffwechsels von einzelnen Muskeln und Organen an lebenden Probanden möglich. Die als ${ }^{31} \mathrm{P}-\mathrm{NMR}$-Spektroskopie bezeichnete Technik beruht ebenfalls auf dem Prinzip der magnetischen Kernspinresonanz, d. h. durch Einstrahlung elektromagnetischer Wellen mit kernspezifischer Frequenz werden die in einem Magnetfeld befindlichen Phosphoratomkerne veranlasst, ihrerseits elektromagnetische Wellen auszustrahlen. Die Resonanzfrequenz ist nicht nur für verschiedene Atomkerne spezifisch; auch bei Atomkernen desselben Isotops sind Verschiebungen der Resonanzfrequenz zu beobachten, die charakteristisch für die chemische Umgebung sind, in der sich diese Atomkerne befinden. Die chemische Verschiebung wird vor allem durch eine mehr oder weniger starke Abschirmung des externen Magnetfeldes an verschiedenen Orten im Molekül durch die Elektronen verursacht. Eine Bestimmung der unterschiedlichen Resonanzfrequenzen der Phosphoratomkerne erlaubt es deshalb, zwischen den einzelnen Stoffwechselprodukten zu unterscheiden. Die chemische Verschiebung wird im allgemeinen relativ zu einem Referenzsignal angegeben. In einem ${ }^{31} \mathrm{P}$-Spektrum der Muskulatur oder des Gehirns eignet sich dafür das Signal von Kreatinphosphat, da dessen Resonanzfrequenz unabhängig vom intrazellulären $\mathrm{pH}$ ist. Die einfachste Methode, lokalisierte Spektren zu erhalten, besteht in der Verwendung einer Oberflächenspule, die direkt über dem Untersuchungsgebiet auf der Oberfläche fixiert wird. Das Gewebevolumen, das zum Spektrum beiträgt, ist im wesentlichen auf das Volumen einer Halbkugel unterhalb der Spule beschränkt. Durch eine Veränderung der Energie der Sendeimpulse lässt sich die Empfindlichkeitscharakteristik der Spule an die Lage und Form des Untersuchungsvolumens anpassen. Mit Hilfe dieser Technik können Veränderungen der zentralen Metaboliten der intrazellulären Energieproduktion - das anorganische Phosphat (Pi), das Kreatinphosphat (PCr) und $\alpha$-, $\beta$-, und $\gamma$-ATP - quantitativ erfasst werden.

3.

Schlachtkörperzusammensetzung

In vivo Studien

In verschiedenen Experimenten wurden Methoden entwickelt, um die Körperzusammensetzung von Schweinen, Schafen und Wassergeflügel zu bestimmen. In einer Studie an Schweinen der Deutschen Landrasse wurden der Muskel- und Fettanteil von Tieren unterschiedlicher Gewichtsgruppen (20,50 und $90 \mathrm{~kg}$ Lebendgewicht) geschätzt (BAULAIN et al., 1996). Die tomographische Untersuchung erfolgte an fünf aussagekräftigen Positionen: Schulter, Brust, Lende, Hüfte und Schinken. Nach der Tomographie wurden die Schweine geschlachtet und zur Kontrolle in die Gewebefraktionen Muskel, Fett und Knochen zerlegt. Die Auswertung der MR-Bilder erfolgte mit Hilfe einer speziellen Bildanalysetechnik (SCHOLZ et al., 1993). Tabelle 1 weist die Genauigkeit der Schätzung anhand der Bestimmtheitsmaße und Schätzfehler aus. Es ist klar ersichtlich, dass die MR-Tomographie eine sehr präzise Schätzung der Muskelund Fettmenge in jeder Gewichtsgruppe ermöglicht. Ebenfalls hohe Schätzgenauigkei- 
ten ergaben sich für die Merkmale Muskel- und Fleischanteil in der mittleren und schweren Gewichtsgruppe. Die Gewebeanteile der im Mittel $20 \mathrm{~kg}$ schweren Tiere ließen sich mit einer etwas eingeschränkten Genauigkeit bestimmen. Mögliche Erklärungen hierfür sind die im Vergleich zu den schwereren Tieren geringere Varianz, aber auch die noch nicht so deutliche Gewebedifferenzierung.

Tabelle 1

Genauigkeit der Schătzung von Gewebemengen und -anteilen bei lebenden Schweinen verschiedener Gewichtsklassen (Accuracy of estimation of body composition in live pigs of different weight groups)

\begin{tabular}{lccccccccc}
\hline & & $20 \mathrm{~kg}$ & & \multicolumn{3}{c}{$50 \mathrm{~kg}$} & & \multicolumn{2}{c}{$90 \mathrm{~kg}$} \\
& $\mathrm{R}^{2}$ & $\mathrm{SEE}$ & $\mathrm{SEE} / \mathrm{s}$ & $\mathrm{R}^{2}$ & $\mathrm{SEE}$ & $\mathrm{SEE} / \mathrm{s}$ & $\mathrm{R}^{2}$ & $\mathrm{SEE}$ & $\mathrm{SEE} / \mathrm{s}$ \\
\hline Muskelmenge [g] & 0,91 & 190 & 0,32 & 0,96 & 265 & 0,20 & 0,89 & 612 & 0,36 \\
Fettmenge [g] & 0,89 & 90 & 0,35 & 0,97 & 150 & 0,19 & 0,91 & 374 & 0,34 \\
Muskelanteil [\%] & 0,55 & 1,46 & 0,73 & 0,83 & 0,92 & 0,46 & 0,87 & 1,19 & 0,38 \\
Fettanteil [\%] & 0,68 & 1,06 & 0,59 & 0,80 & 0,97 & 0,46 & 0,89 & 1,01 & 0,36 \\
\hline
\end{tabular}

$\mathrm{R}^{2}$ : BestimmtheitsmaB; SEE: Schatzehler; s: Standardabweichung

In einer vergleichbaren Studie wurden von Lämmern verschiedener Fleischrassen und deren Kreuzungen mit Finnschafen mit Hilfe der MR-Tomographie an den Positionen Schulter, Brust, Lende und Keule Querschnittsbilder erfasst, um Schätzfunktionen zur Bestimmung der geweblichen Zusammensetzung zu etablieren (STREITZ et al., 1995). Die Vollzerlegung des Schlachtkörpers in Muskelfleisch, Fett und Knochen diente auch hier als Referenz. Tabelle 2 weist die Genauigkeit der Schätzung für die in zwei Gewichtsgruppen unterteilten Tiere aus. Auch hier sind die Gewebemengen mit hoher Genauigkeit $\left(R^{2}>0,9\right)$ zu schätzen; dies gilt auch für den Fleisch- und Fettanteil der schwereren Tiere.

Tabelle 2

Genauigkeit der Schätzung von Gewebemengen und -anteilen bei lebenden Lämmern verschiederier Gewichtsklassen (Accuracy of estimation of body composition in live lambs of different weight groups)

\begin{tabular}{lcccccc}
\hline & \multicolumn{3}{c}{ LGW $\leq 30 \mathrm{~kg}$} & \multicolumn{3}{c}{ LGW $>30 \mathrm{~kg}$} \\
& $\mathrm{R}^{2}$ & $\mathrm{SEE}$ & $\mathrm{SEE} / \mathrm{s}$ & $\mathrm{R}^{2}$ & $\mathrm{SEE}$ & SEE/s \\
\hline Muskelmenge [g] & 0,96 & 160 & 0,20 & 0,91 & 261 & 0,32 \\
Fettmenge [g] & 0,96 & 84 & 0,22 & 0,94 & 195 & 0,26 \\
Muskelanteil [\%] & 0,78 & 1,57 & 0,49 & 0,91 & 1,60 & 0,32 \\
Fettanteil [\%] & 0,86 & 1,49 & 0,39 & 0,90 & 1,64 & 0,34 \\
\hline
\end{tabular}

R2: Bestimmtheitsmaß; SEE: Schaizfehler; s: Standardabweichung

In Abbildung 1 ist anhand des Schätzfehlers (SEE = standard error of estimation) dargestellt, mit welcher Genauigkeit der Fleischanteil des Gesamtkörpers aus einzelnen Aufnahmen in verschiedenen Körperregionen geschătzt werden kann. Zur Orientierung ist im linken Säulenpaar die Standardabweichung des Merkmals angegeben. Es wird deutlich, dass Bilder aus dem Schulterbereich zu einer besseren Schätzung fuhrten als Bilder aus dem Keulen- und Lendenbereich. Eine Kombination mehrerer Messstellen führte zu einer deutlichen Reduktion des Schätzfehlers, der für beide Gewichtsgruppen unter $2 \%$ lag.

Vorteil einer Anwendung von Schätzfunktionen ist, dass anhand weniger Schnittbilder 


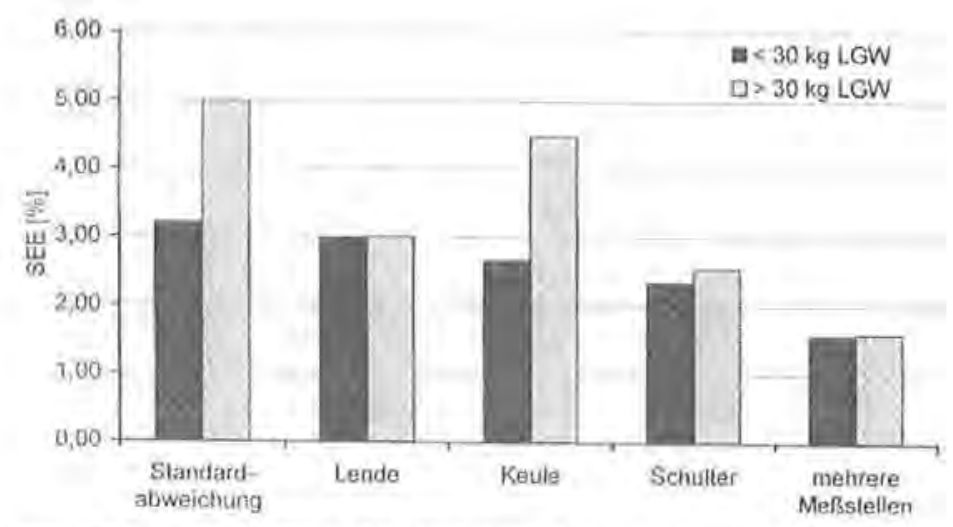

Abb, L: Genauigkeit der Schătzung des Fleischanteils von Lämmern anhand von Querschnittsbildern verschicdener Körperregionen (Accuracy of estimation of body composition in lambs from transverse images of different body regions)

eine Bestimmung der geweblichen Zusammensetzung des Körpers mit hoher Genauigkeit möglich ist. Diese Präzision wird im wesentlichen davon bestimmt, wie gut die im Bild enthaltenen Informationen mit der aufgenommenen Körperregion übereinstimmen und wie aussagekräftig die gewählte Messstelle für die Zusammensetzung des gesamten Körpers ist. Die Nachteile der Schätzung der Körperzusammensetzung aus Bildern bestimmter Regionen sind darin zu sehen, dass das mögliche Informationsspektrum tomographischer Verfahren wie CT und MRT nicht optimal genutzt wird und dass Schätzfunktionen ,gepflegt" werden müssen, d. h. in bestimmten Zeitabständen an das sich verändernde Tiermaterial anzupassen sind. Dazu kommt, dass die Schätzung eines Merkmals mit Hilfe einer Formel nicht unverzerrt ist (z. B. Rassenbias).

Eine unverzerrte Schätzung von Gewebe- oder Organvolumina ist dagegen bei Anwendung einer aus der Anatomie bekannten stereologischen Methode möglich, die u.a. von ROBERTS et al. (1993) verwendet wurde. Dieses Verfahren, das von dem italienischen Mathematiker Bonaventura Cavalieri schon im 17. Jahrhundert beschrieben wurde, setzt voraus, dass der zu quantifizierende Körper über einen Satz paralleler Querschnitte in äquidistantem Abstand vollständig erfasst wird. Prädestiniert zur Aufnahme solcher Querschnitte sind die tomographischen Techniken CT und MRT. Durch Bestimmung der Flächen des interessierenden Organs oder Gewebes in jedem Querschnitt und der Multiplikation der Summe dieser Flächen mit dem Abstand der Schnittbilder ergibt sich das jeweilige Gewebe- oder Organvolumen. Nach THOMPSON und KINGHORN (1992) ist eine Serie von 20 bis 25 CT-Scans ausreichend, um die Zusammensetzung eines Körpers exakt zu erfassen.

In Anlehnung an diese Methode wurde von WIEDERHOLD (1996) das Brustmuskelvolumen an lebenden Enten und Gänsen bestimmt. Über die Aufnahme eines Satzes von parallelen Querschnittsbildern, die den gesamten $M$. pectoralis erfassten, und einem anschließenden Planimetrieren der Brustmuskelflächen in den einzelnen Bildern wurde das Volumen dieses für die Selektion auf Fleischleistung entscheidenden Merkmales errechnet. Ebenso wurden das Volumen der Keulenmuskulatur und des Ab- 
dominalfettes erfasst. Tabelle 3 zeigt die Korrelationen zwischen den mit MRT bestimmten Volumina und den mit Hilfe der Zerlegung erhobenen Teilstückgewichte. Die Korrelationen für den Brustmuskel lagen mit $\mathrm{r}>0,96$ in einem sehr hohen Bereich. Etwas geringere Beziehungen wurden fur die Keulenmuskulatur und das Abdominalfet gefunden. Für diese Körperregionen erwies sich die Abgrenzung vom umliegenden Gewebe als nicht so klar ersichtlich wie beim Brustmuskel.

Tabelle 3

Korrelationen zwischen Muskel- und Fettvolumina aus MRT und Zerlegung fur verschiedene Wassergeflugelspezies (Correlations between muscle and fat volume in different water fowl species determined by means of MRT and total dissection)

Brustmuskel Keulenmuskel Abdominalfett

\begin{tabular}{lllllll} 
Spezies & $\mathrm{n}$ & $\mathrm{r}$ & $\mathrm{n}$ & $\mathrm{r}$ & $\mathrm{n}$ & $\mathrm{r}$ \\
\hline Pekingente & 68 & 0,96 & 63 & 0,87 & 37 & 0,78 \\
Moschusente & 68 & 0,97 & 70 & 0,97 & 64 & 0,82 \\
Mularde & 78 & 0,98 & 77 & 0,84 & 73 & 0,84 \\
Gans & 72 & 0,96 & 70 & 0,80 & 64 & 0,84 \\
\hline
\end{tabular}

In einer Studie an Kaninchen stellten KÖVER et al. (1996) die Eignung der MRT zur Bestimmung des Nierenfettes heraus. Korrelationen zwischen dem in vivo bestimmten Nierenfettvolumen und dem nach der Schlachtung erfassten Gewicht des die Nieren umgebenden Fettes lag bei $r=0,93$ bzw. $r=0,94$.

\section{Post mortem Studien}

In früheren Untersuchungen hatte sich die MRT auch als geeignetes Verfahren zur Bewertung von Schlachtkörpern und Teilstücken erwiesen. Für die Schätzung des Fleischanteiles im Schweineschlachtkörper gab GRIEP (1991) ein Bestimmtheitsmaß von $\mathrm{R}^{2}=0,96$ an. An der Hochrippe vom Rind schätzten BAULAIN et al. (1991) den Fleischanteil mit einer Genauigkeit von ein $\mathrm{R}^{2}=0,80$. BRANSCHEID et al. (1989) benutzten die MRT als Referenz für die Messung der Rückenspeck- und Muskeldicke mit Ultraschall. Die Korrelationen zwischen MR- und US-Messungen lagen mit $\mathrm{r}=$ 0,96 für die Speck- und $r=0,90$ für die Muskeldicke auf einem sehr hohen Niveau.

Für die züchterische Bearbeitung der Bauchzusammensetzung beim Schwein, der aufgrund von Besonderheiten in der Vermarktung ein erhöhtes Interesse der Produzenten gilt, muss der Fleischanteil dieses Teilstückes exakt erfasst werden. Im Rahmen der stationären Geschwister-/Nachkommenprüfung erfolgt dies mit Hilfe einer Schätzformel (THOLEN et al., 1998), in die verschiedene Schlachtkörpermaße wie Speckdicken und Muskelflächen eingehen. Alternativ oder ergänzend kann der Fleischanteil auch durch die planimetrische Auswertung von Video- oder Digitalbildern, die am Anschnitt der 13,/14. Rippe aufgenommen werden, bestimmt werden.

Vor diesem Hintergrund wurde die MR-Tomographie eingesetzt, um den Fleischanteil von insgesamt 197 Schweinebäuchen der Herkünfte Piétrain (Pi), Piétrain x Deutsche Landrasse (Pi $x$ DLS) und Deutsche Landrasse (DLS) volumetrisch zu erfassen (BAULAIN et al., 1998). Dazu wurde eine Aufnahmetechnik verwendet, die die gleichzeitige Erfassung mehrerer paralleler Scheiben ermöglicht. Aus der Summe der 
Flächen, multipliziert mit dem Scheibenabstand, wurden die Muskel- und Fettvolumina des gesamten Bauches und schließlich der Fleisch- und Fettanteil (Vol.-\%) berechnet. Nach der Tomographie wurden die Bäuche grobgeweblich in Fleisch, Fett, Knochen und Schwarte zerlegt.

In Tabelle 4 sind die Korrelationen zwischen den Merkmalen der Bauchzusammensetzung, erfasst durch grobgewebliche Zerlegung einerseits und mit Hilfe der MR-Tomographie andererseits, wieder gegeben. Beim Fleischanteil der Herkünfte DLS und Pi x DLS ergaben sich mit $r=0,93$ und $r=0,94$ sehr hohe Korrelationen. Bei den PiétrainSchweinen war mit $r=0,72$ eine etwas geringere Übereinstimmung zu verzeichnen.

Tabelle 4

Korrelationen zwischen den Merkmalen der Bauchzusammensetzung, erfaßt durch grobgewebliche Zerlegung und MR-Tomographie (Correlations between belly composition traits from dissection and MR tomography)

\begin{tabular}{llccc}
\hline & & Pi & Pix DLS & DLS \\
Zerlegung & MR-Tomographie & & & \\
\hline Gesamtgewicht & Gesamtvolumen & 0,96 & 0,99 & 0,99 \\
Muskelgewicht & Muskelvolumen & 0,89 & 0,95 & 0,94 \\
Fettgewicht & Fettvolumen & 0,92 & 0,97 & 0,96 \\
Fleischanteil (Gew.-\%) & Fleischanteil (Vol.-\%) & 0,72 & 0,94 & 0,93 \\
\hline & & $\mathrm{N}=67$ & $\mathrm{n}=68$ & $\mathrm{n}=62$ \\
\hline
\end{tabular}

Diese Ergebnisse zeigen insgesamt eine sehr hohe Übereinstimmung des durch MRTomographie bestimmten Fleischanteils im Schweinebauch mit dem durch grobgewebliche Zerlegung ermittelten Fleischanteil. Dies gilt vor allem für die Mutterlinie DLS, aber auch für die Kreuzungstiere. Die schlechtere Übereinstimmung zwischen MRI und Zerlegung bei den mageren Bäuchen der Vaterlinie Piétrain könnte auf die in diesem Falle nicht optimale Bildauflösung zurückgeführt werden. Die Größe der Bildpunkte ist vermutlich nicht ausreichend, um eine genauere Differenzierung zwischen Muskel- und Fettgewebe zu erreichen. Aber auch die manuelle Zerlegung ist nicht frei von Ungenauigkeiten, insbesondere dann, wenn sehr dünne Gewebsschichten separiert werden müssen.

An denselben Daten wurde anschließend berechnet, wie exakt die an einem Querschnittsbild des Bauches erfassten Muskel- und Fettflächen das gesamte Teilstück im Vergleich zu den LPA-Maßen schätzen. Aus Abbildung 2 wird anhand des Bestimmtheitsmaßes ersichtlich, dass eine Kombination der konventionellen LPA-Schlachtkörpermaße mit der am Bauch im Bereich der 13./14. Rippe gemessenen Fleisch- und Fettfläche zu einer Erhöhung der Schätzgenauigkeit führt. Die Einzelbetrachtung der Herkünfte macht auch hier deutlich, dass die Schätzung für die Kreuzungstiere und für die Schweine der Mutterlinie präziser ist als für die mageren Piétrain-Schweine.

Ebenfalls an oben beschriebenen Material untersuchten SCHREINEMACHERS et al. (1999), wie genau der Fleischanteil des Bauches über lineare Maße, die direkt an den MR-Bildern des Bauches erfasst wurden, geschätzt werden kann. Die zusätzliche Berücksichtigung einer inneren und äußeren Speckdicke sowie der Dicke des gesamten Bauches aus einem Schnittbild in Höhe des 11./12. Brustwirbels zu den LPA-Maßen erbrachte auch hier eine Erhöhung der Schätzgenauigkeit. Für die Mutterline DL und die Kreuzung Pi x DL wurden Bestimmtheitsmaße von $\mathrm{R}^{2}=0,82$ bzw, 0,76 erreicht. 
Für die mageren Piétrains ergab sich erwartungsgemäß ein niedrigerer Wert von $\mathrm{R}^{2}=$ 0,60 .

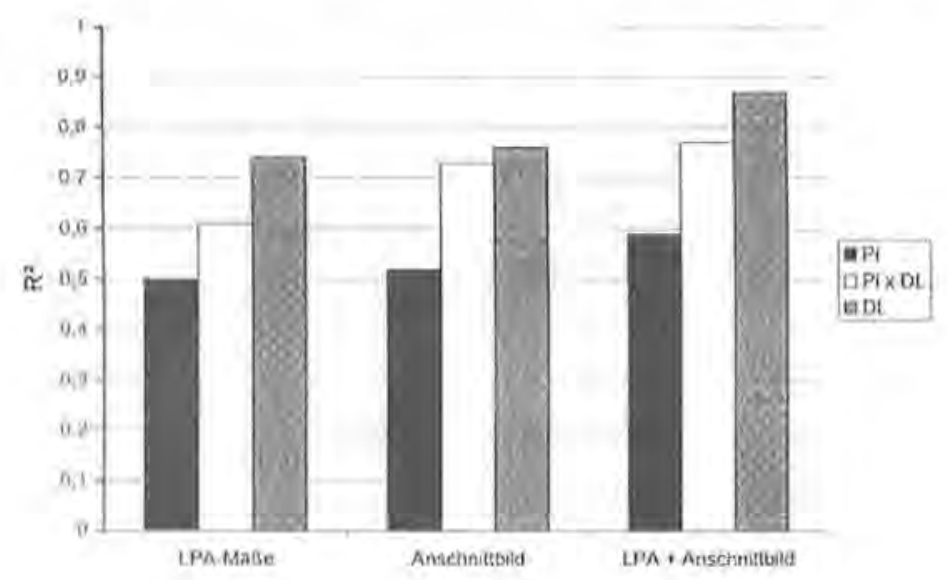

Abb, 2: Genauigkeit der Schătzung des Fleischanteils im Bauch anhand von LPA-Maßen und/oder Muskel- und Fettflächen bestimmt am Bauchbild (Estimation accuracy for percentage of lean from carcass measures and/or muscle and fat areas determined from an image of the belly)

In einer weiteren Studie zum Thema „objektive Bauchbewertung“ wurde die genetische Variation im Bauchfleischanteil von Schweinen aus Mutterlinien untersucht (BAULAIN und HENNE, 1999). Diese Untersuchung sollte durch eine gezielte Auswahl von Vätern und Nachkommen einen Anhaltswert für die Varianz zwischen Vätern liefern. Unter Berïicksichtigung einer relativ geringen Tierzahl und der damit verbundenen Überschätzung der Intraklasskorrelationen deutete sich an, dass im Bauchfleischanteil eine größere Variation zwischen Vätern vorhanden ist als bei den anderen in der Leistungsprüfung erhobenen Schlachtkörpermerkmalen. Dies gilt insbesondere für die Rückenspeckdicke, die im Rahmen der Selektion auf Fleischfülle über Ultraschallmessungen schon lange intensiv züchterisch bearbeitet wird. Eine gesonderte Rangierung der Väter nach dem Bauchfleischanteil könnte demnach ein geeignetes Mittel sein, dieses Teilstück gezielt züchterisch zu bearbeiten.

Bei Masthähnchen wurde die MRT als Referenz verwendet, um die optimalen Messstellen für den Einsatz von Ultraschallgeräten zur Quantifizierung des Brustmuskels zu finden (DAVENEL et al., 1999).

\subsection{Fleischqualität und Stressanfälligkeit}

Eine erhöhte Stressanfälligkeit beim Schwein hat Mängel in der Fleischbeschaffenheit und erhöhte Ausfälle vor der Schlachtung zur Folge. Ursachen sind sowohl genetische als auch umweltbedingte Störungen im Energiestoffwechsel des Muskelgewebes. Die lang andauernde Selektion auf Fleischfülle führte zu einer Bevorzugung stressempfindlicher Schweine in der Züchtung. Um diesem Merkmalsantagonismus entgegenzuwirken, wurden die Zusammenhänge zwischen Muskelphysiologie, Stressanfällig- 
keit und Fleischbeschaffenheit intensiv untersucht und unter Anwendung geeigneter Selektionskriterien in der Züchtung berücksichtigt. Mit Hilfe des von FUJII et al. (1991) vorgestellten MHS-Gentests können homozygot negative (NN), heterozygote $\mathrm{Nn}$ ) und homozygot positive (nn) Genotypen sicher unterschieden werden. Dieser Test löste den bis dahin eingesetzten Halothantest ab, der darauf basierte, dass das Narkosegas Halothan bei stressempfindlichen Schweinen (nn) die maligne Hyperthermie induziert.

Die ${ }^{31}$ P-NMR-Spektroskopie ermöglichte erstmals die nicht invasive, kontinuierliche Beobachtung der zellulären Stoffwechselvorgänge am lebenden Tier und gab weiteren Aufschluss über muskelphysiologische Abläufe beim Schwein in Abhängigkeit vom MHS-Genotyp (DECANNIERE et al., 1993; SCHOLZ et al., 1995). Studien am Schlachtkörper und in vitro erweiterten die Kenntnisse über Abbauvorgänge in der Muskulatur, die im Zusammenhang mit der Fleischreifung von Bedeutung sind (MOESGARD et al., 1993; LAHUCKY et al., 1993; LAHUCKY et al., 1998). In allen Untersuchungen unterschieden sich die stressempfindlichen Tiere (nn) deutlich von den stressresistenten Genotypen NN und $\mathrm{Nn}$, wobei die heterozygoten $\mathrm{z}$. T. eine intermediäre Position einnahmen.

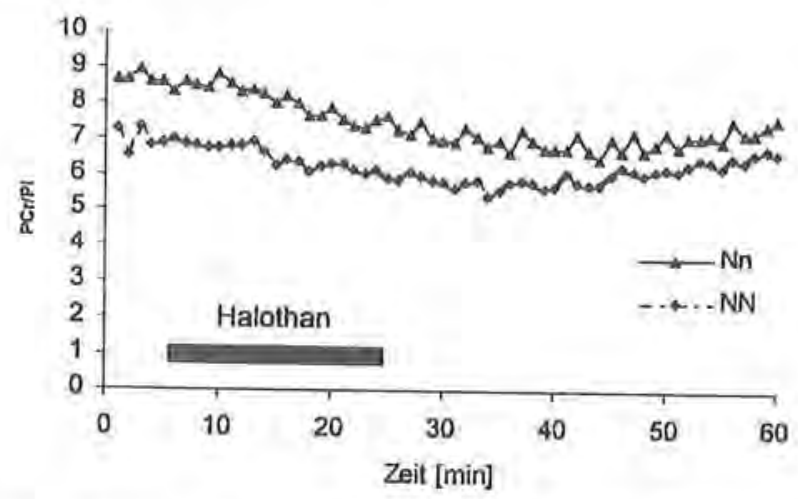

Abb. 3: Verlauf des Quotienten aus PCr/Pi vor, während und nach der Halothanbelastung bei Schweinen der Genotpypen NN und $\mathrm{Nn}(\mathrm{PCr} / \mathrm{Pi}$ before, during and after halothane administration in pigs of $\mathrm{NN}$ and $\mathrm{Nn}$
genotype)

In der von KOHN et al. (1998) vorgestellten Studie wurde die ${ }^{31}$ P-NMR-Spektroskopie erstmals an einem Ganzkörpertomographen eingesetzt, um muskelphysiologische Reaktionen von insgesamt 35 Schweinen unterschiedlichen MHS-Genotyps unter Halothanbelastung zu erfassen. Neben lebenden Schweinen wurden auch Schlachtkörper untersucht, um die quantitativen Veränderungen der Phosphormetaboliten post mortem zu beobachten. Die in der in-vivo Untersuchung gewählte Halothanbelastung von 2,0 Vol,-\% führte zu keiner erkennbaren Reaktion im Energiestoffwechsel der Muskulatur von NN- und Nn-Genotypen (Abb. 3). Tendenziell war jedoch ein leichtes Absinken des $\mathrm{PCr} / \mathrm{Pi}$-Verhältnnisses nach Beginn der Halothanzufuhr $(\mathrm{t}=5 \mathrm{~min}) \mathrm{zu}$ beobachten, um nach Beendigung der Belastungsphase $(t=25$ min) wieder auf das Ausgangsniveau anzusteigen. Deutliche Reaktionen in Form eines starken Abfalls der PCr-Kon- 
zentration und einem entsprechenden Anstieg der Pi-Konzentration zeigten erwartungsgemäß die nn-Genotypen. Klare Unterschiede zwischen den NN- und Nn-Genotypen wurden jedoch beim postmortalen Verlauf von $\mathrm{PCr}$ - und $\mathrm{Pi}$-Anteilen sowie des aus den Spektren berechneten $\mathrm{pH}$-Wertes im M. biceps femoris beobachtet.

4.

\section{Schlussfolgerungen}

Für die Tierzuchtforschung bieten die nicht invasiven Verfahren MR-Tomographie und -Spektroskopie sehr vielfältige und außergewöhnliche Anwendungsmöglichkeiten. In Kombination mit speziellen Bildauswertungsmethoden ermöglicht die MRT eine äußerst genaue Bestimmung der Körperzusammensetzung am lebenden Tier und am Schlachtkörper. Über wiederholte Messungen derselben Probanden ist eine Analyse des individuellen Wachstums von Geweben, Körperpartien, einzelnen Muskeln und Organen möglich. Ein weiterer positiver Aspekt ist, dass bei dieser Vorgehensweise eine geringere Anzahl von Tieren im Versuch ausreicht als bei herkömmlichen Stufenschlachtungen mit anschließender grobegeweblicher Zerlegung von Geschwistertieren. Aufgrund der exakten Trennung von Weichgeweben und der Möglichkeit einer direkten Messung von Gewebevolumina kann die MR-Tomographie statt der sehr zeit- und arbeitsaufwendigen grobgeweblichen Zerlegung als Referenzmethode zur Bestimmung des Körperzusammensetzung dienen. Von dieser Möglichkeit wurde bci der objektiven Bewertung von Schweinebäuchen im Rahmen der Schlachtleistungsprüfung bereits Gebrauch gemacht. Eine Verwendung der MRT als Referenzmethode für die Leistungsprüfung könnte in Zukunft eine schnellere Anpassung von Schätzformeln zur Bestimmung des Fleischanteils oder Handelswertes von Schlachtkörpern ermöglichen und bei der Weiterentwicklung von Verfahren, die in der praktischen Tierzucht benötigt werden - wie z. B. Ultraschall und Videobildanalyse - hilfreich sein. Darüber hinaus ist die MRT aber auch für diagnostische Zwecke einsetzbar, beispielsweise bei der Beurteilung von haltungsbedingten Erkrankungen des Bewegungsapparates.

Die spezielle Möglichkeit, in einem MRT-Gerät ab einer bestimmten Magnetfeldstärke auch spektroskopische Untersuchungen durchzuführen, erweitert das Anwendungspotential der Magnet-Resonanz für die Tierzuchtforschung erheblich. So kann durch die ${ }^{31}$ P-NMR-Spektroskopie eine Online-Erfassung der Phosphormetaboliten des Muskelstoffwechsels auf nicht invasivem Wege erfolgen. Sie ist daher vorzüglich für die weitere Aufklärung möglicher muskelphysiologischer Unterschiede zwischen verschiedenen Genotypen geeignet.

MRT und MRS haben sich aufgrund dieser vielfältigen Anwendungsmöglichkeiten für die Tierzuchtwissenschaft und speziell für die Fleischforschung als sehr wertvolle Techniken erwiesen, die im Rahmen anwendungsorientierter Forschungsarbeiten zu Qualitätssicherung und Prozesskontrolle eingesetzt werden.

\section{Literatur}

BAULAIN, U.; HENNE, H.: Variation des Fleischanteiles im Bauch von Schweinen aus Mutterlinien. Arch. Tierz, Dummerstorf 42 (1999), 431-440 
BAULAIN, U.; HENNING, M; KALLWEIT, E.:

Bestimmung der Korperzusammensetzung von Landrasse-Schweinen unterschiedlichen Alters mittels MRI. Arch. Tierz., Dummerstorf 39 (1996), 43I-440

BAULAIN, U.; HENNING, M; THOLEN, E.; WITTMANN, W.; PESCHKE, W.:

Objektive Erfassung des Fleischanteils im Schweinebauch. 2. Mitt:: Verwendung von Bildinformationen aus dem MR-Imaging. Zuchtungskunde, Stuttgart 70 (1998), 205-212

BAULAIN, U.; SCHWERDTFEGER, R.; KALM, E.:

Composition of carcass cuts assessed by Magnetic Resonance Tomography (MRT). Proc. 42nd Annual Meeting European Association for Animal Production (EAAP), Berlin, 08.-12.09.1991

BRANSCHEID, W; SACK, E.; KALLWEIT, E.; HÖRETH, R.: BAULAIN, U.:

Non-invasive methods in pig grading: A reliable possibility for calibration of grading devices. Proc. 35 th International Congress of Meat Science and Technology, Kopenhagen, 20.-25.08.1989, 239-243

DAVENEL, A.; MARTY-MAHE, P; COLLEWET, G.; LOISEL, P: Research at CEMAGREF on vision and NMR/MRI techniques for improving meat grading and classification. Proc. European Expert Colloquium on Grading \& Classification, Kulmbach, 15.06.1999, 38-42

DECANNIERE, C; , VAN HECKE, P.; VANSTAPEL, F.; VILLE, H.; GEERS, $R$.: Metabolic response to halothane in piglets susceptible to malignant hyperthermia. An in vivo ${ }^{31} \mathrm{P}-\mathrm{NMR}$ study. J. Appl. Physiol. 75 (1993), 955-962

FUII, J; OTSU, K.; ZORZATO, F; DE LEON, S.; KHANNA, V.K.; WELLER, J.; O'BRIEN, P.J; MACLENNAN, D,H.:

Identification of a mutation in the porcine ryanodine receptor that is associated with malignan hyperthermia. Science, Washington 253 (1991), 448 -451

GRIEP, W:

Schătzung der grobgeweblichen Schlachtkðrperzusammensetzung von Schweinen unterschiedlichen Alters mit der Magnet-Resonanz-Tomographie (MRT). Univ, Gottingen, Diss., 1991, 118 S.

KALLWEIT, E.; WESEMEIER, H. H.; SMIDT, D.; BAULAIN, U.:

Einsatz der Magnet-Resonanz-Messung in der Tierzuchtforschung. Arch. Tierz., Dummerstorf 37 (1994), $105-120$

KÖVER, G.; SORENSEN, P.; SZENDRÖ, Z.; MILISITS, G.;

In vivo measurement of perinatal fat by magnetic resonance tomography. Proc. 6th World Rabbil Congress, Vol. 3, Toulouse, 1996

KOHN, G.; BAULAIN, U; HENNING, M.; LAHUCKY, R.; LEIBERITZ, D.; KALLWEIT, E. In vivo und post mortem Untersuchungen des Muskelstoffwechseis von Schweinen mit unterschiedlichem MHS-Genotyp mit Hilfe der ${ }^{31}$ P-NMR-Spektroskopie. Arch. Tierz., Dummerstorf 4 I (1998), 299-3I0

LAHUCKY, R.; MOJTO, J.; POLTARSKY, J.; MIRI, A.; RENOU, J.P.; TALMANT, A.; MONIN, G.: Valuation of halothane sensitivity and prediction of post-mortem muscle metabolism in pigs from a muscle biopsy using ${ }^{31}$ P NMR spectroscopy, Meat Sci. 33 (1993), 373-384

LAHUCKY, R.; CHRISTIAN, L. L.; KOVAC, L.; STALDER, K. J.; BAUEROVA, M.:

Meat quality assessed ante- and post mortem by different ryanodine receptor gene status of pigs. Meat Sci. 47 (1997), 277-285

MOESGAARD, B.; QUISTORFF, B.; GROSFIELD CHRISTENSEN, V.; JORGENSEN, I.T.; JORGENSEN, P.F.

Differences of post-mortem ATP turnover in skeletal muscle of normal and heterozygote malignant hyperthermia pigs: Comparison of ${ }^{31}$ P-NMR and analytical biochemical measurements. Meat Sci. 39 (1995), 43-57

ROBERTS, N.; CUZ-ORIVE, L. M.; REID, N. M. K.; BRODIE, D. A.; BOURNE, M.; EDWARDS, R. H. T.; Unbiased estimation of human body composition by the Cavalieri method using magnetic resonance imaging. J, of Microsc. 171 (1993), 239-253

SCHOLZ, A.; BAULAIN, U,; KALLWEIT, E.:

Quantitative Analyse von Schnittbildern aus der Magnet-Resonanz-Tomographie. Zuchtungskunde, Stuttgart 65 (1993), 206-215

SCHOLZ, A; MITCHELL, A. D., WANG, P, C; SONG, H; YAN, Z:

Muscle metabolism and body composition of pigs with different ryanodine receptor genotypes studied by means of ${ }^{31} \mathrm{P}$ nuclear magnetic resonance spectroscopy and ${ }^{1} \mathrm{H}$ magnetic resonance imaging. Arch. Tierz., Dummerstorf 38 (1995), 539-552 
SCHREINEMACHERS, H.-J.; THOLEN, E.; BAULAIN, U.; HENNING, M.; TRAPPMANN, W.:

Untersuchungen zur Objektivierung der Bauchbewertung bei Schlachtschweinen unter Verwendung nichtinvasiver Verfahren. Landbauforschung Volkenrode, Sonderheft 193 (1999), 123-128

STREITZ, E.; BAULAIN, U; KALLWEIT, E.:

Untersuchungen zur Korperzusammensetzung wachsender Lämmer mit Hilfe der Magnet-ResonanzTomographie (MRT). Zuchtungskunde, Stuttgart 67 (1995), 392-403

SZABO, CS ; BABINSZKY, L; VERSTEGEN, M.W.A.; VANGEN, O; JANSMAN, A.J.M.; KANIS, E.:

The application of digital imaging techniques in the in vivo estimation of the body composition of pigs: a review. Livest. Prod. Sci. 60 (1999), 1-11

THOLEN, E.; PESCHKE, W.; BAULAIN, U.; SCHELLANDER, K.:

Objektive Erfassung des Fleischanteils im Schweinebauch, 1. Mitt: Entwicklung von Schătzgleichungen aus Schlachtkठrpermaßen. Zuchtungskunde, Stuttgart 70 (1998), 196-204

THOMPSON, J. M; KINGHORN, B,;

CATMAN - A program to measure CAT-scans for prediction of body components in live animals. Proc, of the Austral. Assoc, of Anim. Breed. and Genetics 10 (1992), 5

VANGEN, O; JOPSON, N.:

Research application of non-invasive techniques for body composition. Proc, 47th Annual Meeting European Association for Animal Production (EAAP), Lillehammer, 25.08.-29,08.1996

WIEDERHOLD, S.:

In vivo Messungen ausgewahlter Merkmale der Körperzusammensetzung bei Wassergeflugel mit Hilfe der Magnet-Resonanz-Tomographie (MRT). Univ. Halle, Diss., 1996, 121 S,

Eingegangen: 25.01 .2001

Akzeptiert: 13.02.2001

Anschrift der Verfasser

Dr. ULRICH BAULAIN, Dr. MARTTNA HENNING

Institut für Tierzucht und Tierverhalten der FAL, Mariensee

Holtystraße 10

D-31535 Neustadt 